\title{
Pharmacokinetics of Oxaliplatin in a Hemodialysis Patient with Metastatic Colon Cancer
}

\author{
Yoshiaki Nagatani ${ }^{1}$, Yoshinori Imamura ${ }^{1 *}$, Tsutomu Nakamura ${ }^{2}$, Kazuhiko Yamashita ${ }^{3}$, \\ Mamoru Okuno 3 , Hiroyuki Yasui ${ }^{4}$, Jun Hiraoka ${ }^{4}$, Riho Niigata ${ }^{4}$, Keiji Kono ${ }^{5}$, Yasuko Hyo- \\ go $^{1}$, Hirotaka Suto ${ }^{1}$, Kei Takenaka ${ }^{1}$, Yohei Funakoshi ${ }^{1}$, Masanori Toyoda $^{1}$, Naomi Kiyo- \\ ta $^{1,6}$ and Hironobu Minami ${ }^{1,6}$
}

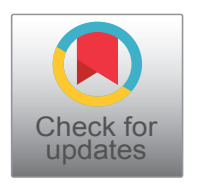

${ }^{1}$ Department of Medical Oncology/Hematology, Kobe University Hospital, Kusunoki-cho, Chuo-ku, Kobe, Japan

${ }^{2}$ Education and Research Center for Clinical Pharmacy, Osaka University of Pharmaceutical Sciences, Nasahara, Takatsuki, Osaka, Japan

${ }^{3}$ Department of Pharmacy, Kobe University Hospital, Kusunoki-cho, Chuo-ku, Kobe, Japan

${ }^{4}$ Department of Analytical and Bioinorganic Chemistry, Kyoto Pharmaceutical University, Misasaginakauchi-cho, Yamashina-ku, Kyoto, Japan

${ }^{5}$ Division of Nephrology and Kidney Center, Kobe University Graduate School of Medicine, Kusunoki-cho, Chuo-ku, Kobe, Japan ${ }^{6}$ Kobe University Hospital Cancer Center, Kusunoki-cho, Chuo-ku, Kobe, Japan

*Corresponding author: Yoshinori Imamura, MD, PhD, Department of Medical Oncology and Hematology and Cancer Center, Kobe University Hospital, 7-5-1, Kusunoki-cho, Chuo-ku, Kobe 650-0017, Japan, Tel: +81-78-382-5820, Fax: +8178-382-5821

\begin{abstract}
Background: Although oxaliplatin is one of the main anticancer agents used against colorectal cancer, data on the pharmacokinetics of oxaliplatin in patients on hemodialysis are limited. In this case study, we evaluated the pharmacokinetics and safety of oxaliplatin in a patient on hemodialysis treated for colon cancer.

Methods: A 68-year-old Japanese woman receiving chronic hemodialysis was treated for metastatic colon cancer with modified FOLFOX6 plus bevacizumab every 2 weeks. Initial oxaliplatin dose was reduced to $42.5 \mathrm{mg} / \mathrm{m}^{2}$, which was $50 \%$ of the standard dose. 4-hour hemodialysis was begun 1.5 hours after the end of oxaliplatin infusion. Serial plasma concentrations of total and free platinum were measured before and until the first 48 hours after oxaliplatin infusion. Adverse events were evaluated using Common Terminology Criteria for Adverse Events version 4.0.

Results: The pharmacokinetics of free platinum showed a bimodal pattern. Maximum concentrations were $0.73,0.80$, and $1.03 \mu \mathrm{g} / \mathrm{mL}$ after a dose of $42.5,42.5$, and $50 \mathrm{mg} / \mathrm{m}^{2}$ oxaliplatin, respectively. The area under the curve in the first
\end{abstract}

cycle was $14.2 \mu \mathrm{g}$ 'h/mL. No clinically meaningful accumulation of free platinum was seen. No new safety signals were identified, and no Grade 2-4 adverse events were observed.

Conclusions: These pharmacokinetics data for oxaliplatin in a hemodialysis patient were comparable with those in patients with normal renal function. Modified FOLFOX6 plus bevacizumab every 2 weeks could be safely given in a hemodialytic colon cancer patient with a reduction in oxaliplatin dose to $50 \mathrm{mg} / \mathrm{m}^{2}$, if hemodialysis was performed 1.5 hours after the end of oxaliplatin infusion.

\section{Keywords}

Oxaliplatin, Hemodialysis, Pharmacokinetics, Colorectal cancer, Modified FOLFOX6 plus bevacizumab

\section{Introduction}

The number of patients with end-stage renal disease is increasing rapidly worldwide. These patients require dialysis treatment or renal transplantation for survival [1]. In Japan, hemodialysis patients have been increas-

Citation: Nagatani Y, Imamura Y, Nakamura T, Yamashita K, Okuno M, et al. (2019) Pharmacokinetics of Oxaliplatin in a Hemodialysis Patient with Metastatic Colon Cancer. Int J Oncol Res 2:017. doi. org/10.23937/2643-4563/1710017

Accepted: September 14, 2019; Published: September 16, 2019

Copyright: (c) 2019 Nagatani Y, et al. This is an open-access article distributed under the terms of the Creative Commons Attribution License, which permits unrestricted use, distribution, and reproduction in any medium, provided the original author and source are credited. 
ing yearly [2], and the survival rate of patients with endstage renal disease has shown a gradual improvement. The risk of cancer is increased in patients on hemodialysis, and the need for chemotherapy in hemodialysis patients is accordingly increasing [3-5].

Worldwide, colorectal cancer was the third-most common malignancy in men $(746,000$ cases; $10.0 \%$ of total cancer) and the second-most common in women (614,000 cases; $9.2 \%$ of total cancer cases) in 2012 [6]. Standard treatments for primary chemotherapy now include the addition of a molecular-targeted agent to oxaliplatin- or irinotecan-based chemotherapy, and mean survival time in patients with recurrent or metastatic colorectal cancer has improved to over 30 months $[7,8]$. Oxaliplatin is eliminated mainly by the kidneys, and its pharmacokinetics are reported to change in patients with renal dysfunction [9]. Although one report noted that a dose reduction is not necessary if creatinine clearance is more than $20 \mathrm{~mL} / \mathrm{min}$ [10], it is not clear whether oxaliplatin can be administered in patients with severe renal dysfunction or hemodialysis. Furthermore, while some reports have described the administration of oxaliplatin to hemodialysis patients [11-13], dosing standards for this population have also not been determined.

Here, we evaluated the pharmacokinetics and safety of oxaliplatin administration in a hemodialysis patient.

\section{Materials and Methods}

A 68-year-old Japanese woman who had been maintained on hemodialysis because of chronic nephritis since 2011 was diagnosed with recurrent colon adenocarcinoma in 2015. She was treated with modified FOLFOX6 plus bevacizumab after irinotecan-based chemotherapy. Initial oxaliplatin dose was $42.5 \mathrm{mg} / \mathrm{m}^{2}$, or $50 \%$ of standard dose for modified FOLFOX6 (oxaliplatin $85 \mathrm{mg} / \mathrm{m}^{2}$ IV over 2 hours plus I-leucovorin $200 \mathrm{mg} / \mathrm{m}^{2}$ IV over 2 hours, followed by 5 -fluorouracil $400 \mathrm{mg} / \mathrm{m}^{2}$ IV bolus, followed by 5 -fluorouracil $2,400 \mathrm{mg} / \mathrm{m}^{2}$ over 46 hours continuous infusion; repeat every 2 weeks). After safety was confirmed, the dose of oxaliplatin was increased to $50 \mathrm{mg} / \mathrm{m}^{2}$. 4-hour dialysis, with a dialysate (Kindaly AF-3), was performed at 1.5 hours after the end of oxaliplatin infusion in accordance with a previous study [11]. Blood flow rate was $220 \mathrm{~mL} / \mathrm{min}$, bicarbonate-buffered dialysate flow rate was $500 \mathrm{~mL} / \mathrm{min}$, and dialysis water removal was $1.7 \mathrm{~L}$. Low molecular weight heparin was infused for anticoagulation. Blood samples for pharmacokinetic evaluation were collected in tubes containing sodium heparin before oxaliplatin infusion; 1 and 2 (end of infusion) hours after the start of oxaliplatin infusion; and 15, $30 \mathrm{~min}, 1,1.5$ (start of hemodialysis), 3.5, 5.5 (end of hemodialysis), 7.5, 22, and 46 hours after the end of infusion. The samples were centrifuged at $1700 \mathrm{~g}$ at $4{ }^{\circ} \mathrm{C}$ for $10 \mathrm{~min}$, and a portion of the plasma was stored in a deep freezer at $-70{ }^{\circ} \mathrm{C}$ until assay of total platinum. The remaining plasma was ultra-filtrated using an ultrafiltration tube (Amicon ${ }^{\circledR}$ filter with a molecular cut-off of $30,000 \mathrm{Da}$ ) at $4{ }^{\circ} \mathrm{C}$ and stored at $-70{ }^{\circ} \mathrm{C}$ until assay of free platinum. Total and free platinum concentrations were analyzed by inductively coupled plasma mass spectrometry (ICP-MS) using an Agilent $7700 \times$ ICP-MS (Agilent Technologies, Inc., Santa Clara, CA, USA) [14], and the area under the curve from 0 to 48 hours $\left(A \cup C_{0-48}\right.$ ) after oxaliplatin infusion was calculated using the linear trapezoidal method. Free platinum concentration of oxaliplatin in our patient was compared with that in historical patients with normal renal function treated with $85 \mathrm{mg} / \mathrm{m}^{2}$ oxaliplatin (Japanese package insert). Adverse events were monitored at least bi-weekly throughout the treatment and evaluated using Common Terminology Criteria for Adverse Events version 4.0.

\section{Results}

The pharmacokinetics of free platinum showed a bimodal pattern (Figure 1). Free platinum concentration reached a peak at the end of infusion and decreased immediately in the next 30 minutes. After a slight increase caused by free platinum moving from tissue to blood, it decreased from the start to the completion of hemodialysis, then increased up to 24 hours and maintained a plateau thereafter.

The pharmacokinetics of total platinum also showed a bimodal pattern (Figure 2). Total platinum concentration reached a peak at 15 minutes after the end of oxaliplatin infusion and decreased immediately in the next 15 minutes. After maintaining a plateau for one hour, it decreased from the start of hemodialysis to 2 hours after the completion of hemodialysis, then increased up to 24 hours and decreased thereafter.

Results for the pharmacokinetic parameters of initial concentration $\left(C_{0}\right)$, maximum concentration $\left(C_{\max }\right)$ and $\mathrm{AUC}_{0-48}$ of plasma total and free platinum are shown in Table 1. Although $\mathrm{C}_{0}$ slightly increased with each successive cycle, none of these increases was clinically significant. Compared with normal renal function, $\mathrm{C}_{\max }$ was slightly lower on administration at $42.5 \mathrm{mg} / \mathrm{m}^{2}$, but slightly higher at $50 \mathrm{mg} / \mathrm{m}^{2}$. Free platinum AUC was 14.2 $\mu \mathrm{g}^{*} \mathrm{~h} / \mathrm{mL}$ at a dose of $42.5 \mathrm{mg} / \mathrm{m}^{2}$ in the first cycle, or about 1.3 times the value obtained from historical patients with normal renal function treated with $85 \mathrm{mg} / \mathrm{m}^{2}$ oxaliplatin. AUC in the second and third cycles could not be calculated because the free platinum concentration after 48 hours could not be measured.

With regard to safety, grade 1 adverse events included nausea and anorexia during the first cycle; nausea, anorexia, and mucositis during the second; and nausea, anorexia, mucositis, diarrhea, and peripheral sensory neuropathy during the third. No grade 2-4 adverse events were observed. Regarding efficacy, a CT scan performed after five cycles of modified FOLFOX6 plus 


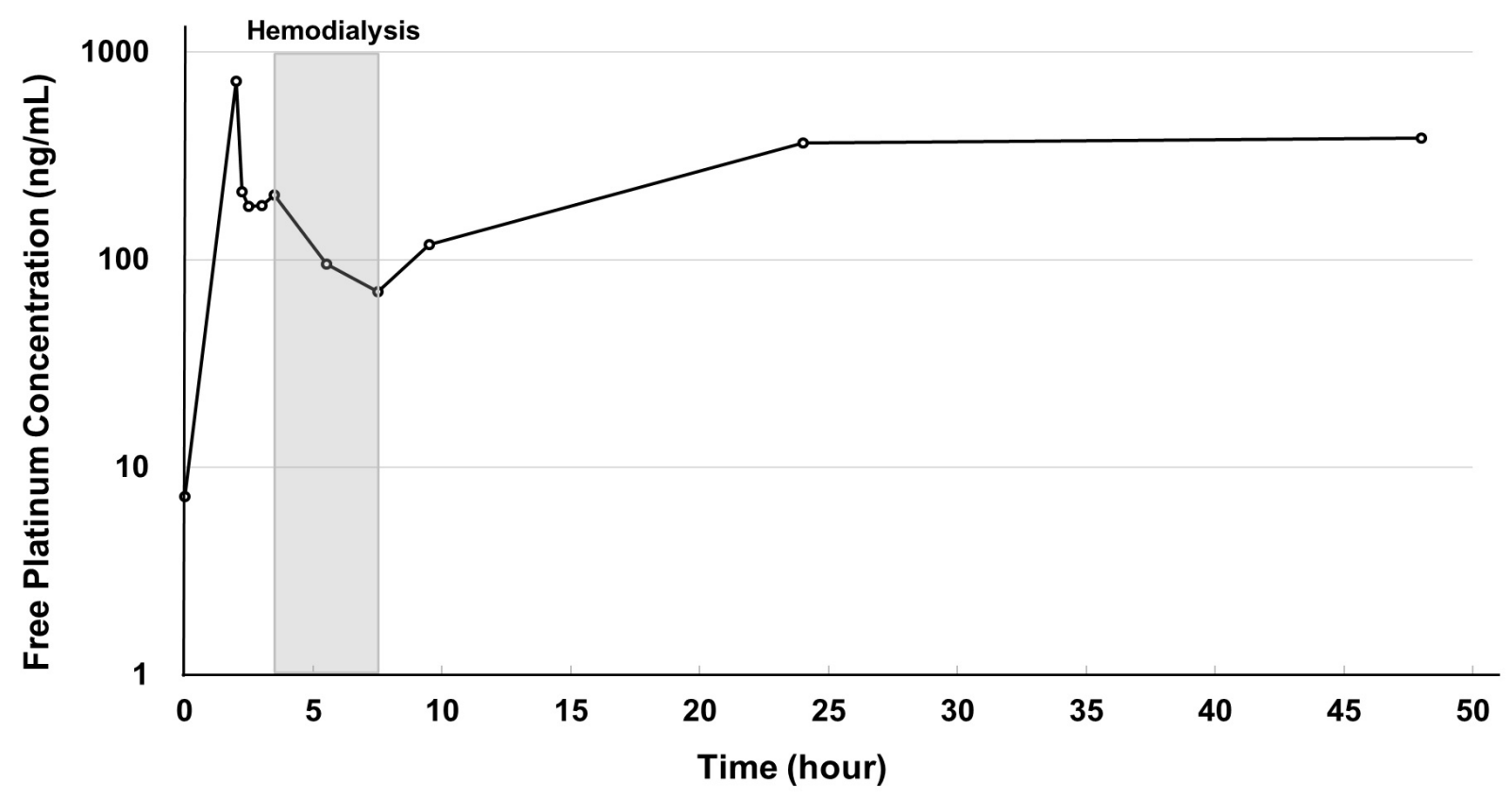

Figure 1: Time curve of plasma free platinum concentration.

Free platinum concentration reached a peak at the end of infusion and decreased immediately in the next 30 minutes. After a slight increase caused by free platinum moving from tissue to blood, it decreased from the start to the completion of hemodialysis, then increased up to 24 hours and maintained a plateau thereafter.

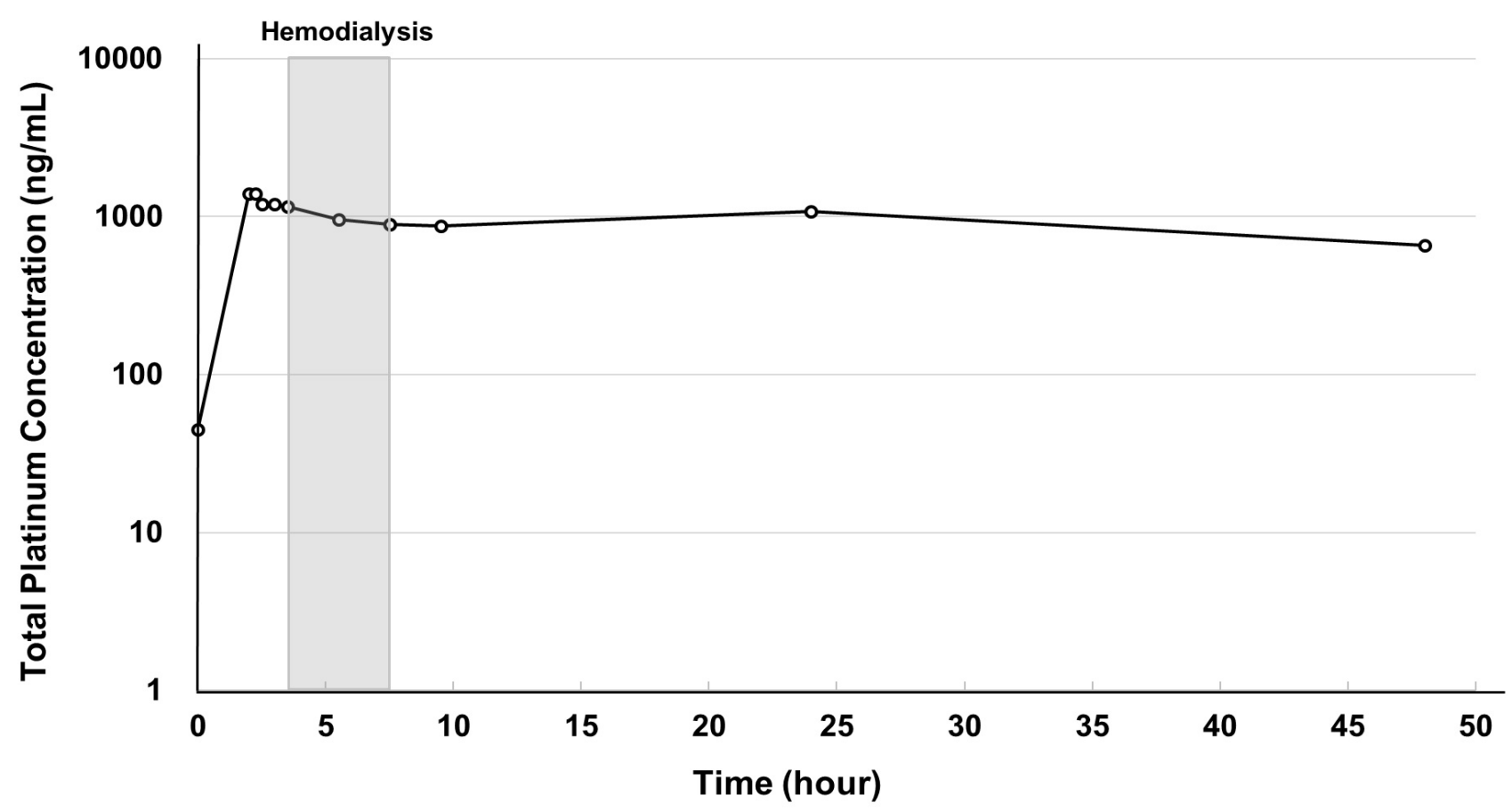

Figure 2: Time curve of plasma total platinum concentration.

Total platinum concentration reached a peak at 15 minutes after the end of oxaliplatin infusion and decreased immediately in the next 15 minutes. After maintaining a plateau for one hour, it decreased from the start of hemodialysis to 2 hours after the completion of hemodialysis, then increased up to 24 hours and decreased thereafter.

bevacizumab was evaluated as progressive disease by RECIST ver1.1.

\section{Discussion}

Modified FOLFOX6 plus bevacizumab every 2 weeks was safely administered to a hemodialysis patient with colon cancer by reducing oxaliplatin dose to $50 \mathrm{mg} / \mathrm{m}^{2}$ and conducting hemodialysis 1.5 hours after the end of oxaliplatin infusion. $\mathrm{AUC}_{0-48}$ of free platinum showed a slightly high value with a bimodal pattern, while $C_{\text {max }}$ showed comparable results to those from patients with normal renal function (Table 1). As for toxicity, only grade 1 adverse event was observed, and no grade 2-4 or new adverse events were observed. 
Table 1: Pharmacokinetic parameters.

\begin{tabular}{|c|c|c|c|c|c|c|}
\hline \multirow[b]{2}{*}{$\begin{array}{l}\text { Cycle } \\
\text { (Oxaliplatin dose) }\end{array}$} & \multicolumn{3}{|c|}{ Total Platinum } & \multicolumn{3}{|c|}{ Free Platinum } \\
\hline & $\begin{array}{l}C_{0} \\
(\mu \mathrm{g} / \mathrm{mL})\end{array}$ & $\begin{array}{l}C_{\max } \\
(\mu g / m L)\end{array}$ & $\begin{array}{l}A_{0-48} \\
\left(\mu g^{*} h / m L\right)\end{array}$ & $\begin{array}{l}C_{0} \\
(\mu \mathrm{g} / \mathrm{mL})\end{array}$ & $\begin{array}{l}C_{\max } \\
(\mu \mathrm{g} / \mathrm{mL})\end{array}$ & $\begin{array}{l}\text { AUC }_{0-48} \\
\left(\mu g^{*} h / m L\right)\end{array}$ \\
\hline $\begin{array}{l}\text { First cycle } \\
\left(42.5 \mathrm{mg} / \mathrm{m}^{2}\right)\end{array}$ & 0 & 1.4 & 44.0 & 0 & 0.73 & 14.2 \\
\hline $\begin{array}{l}\text { Second cycle } \\
\left(42.5 \mathrm{mg} / \mathrm{m}^{2}\right)\end{array}$ & 0.2 & 2.0 & - & 0.02 & 0.80 & - \\
\hline $\begin{array}{l}\text { Third cycle } \\
\left(50 \mathrm{mg} / \mathrm{m}^{2}\right)\end{array}$ & 0.3 & 1.7 & - & 0.04 & 1.03 & - \\
\hline $\begin{array}{l}\text { Normal function } \\
\left(85 \mathrm{mg} / \mathrm{m}^{2}\right)^{*}\end{array}$ & - & - & - & 0 & 0.93 & 10.9 \\
\hline
\end{tabular}

"Data from Japanese package insert;

$\mathrm{C} 0$ : Initial concentration; $\mathrm{C}_{\max }$ : Maximum concentration; $\mathrm{AUC}_{0-48}$ : Area under the curve from 0 to 48 hours.

Oxaliplatin changes into three kinds of active form (dichloro-, monochloro-, and diaquo-diaminocyclohexane platin) capable of interaction when entering the blood. Pharmacokinetics shows three phases, namely $\alpha$ (distribution phase), $\beta$ (elimination phase), $\gamma$ (redistribution phase). Free platinum concentration rapidly decreases in $\alpha$-phase, gradually decreases in $\beta$-phase, and decreases quite gently in $\gamma$-phase $[15,16]$. However, because most free platinum after oxaliplatin infusion is inactive low molecular weight platinum-amino acid conjugates (glutamine, L-methionine, and L-cysteine) capable of renal elimination $[9,16]$ free platinum in $\beta$ - and $\gamma$ - phases is mostly the inactive form.

Consistent with previous reports $[11,12,16]$, plasma free platinum concentration in our patient showed a bimodal pattern. With normal renal function, the second peak caused by free platinum moving from tissue to blood does not appear due to prompt elimination of free platinum by the kidney. In contrast, patients on hemodialysis are unable to eliminate platinum from the kidney, resulting in a second peak which affects the value of AUC. However, since the free platinum at the second peak, corresponding to the $\gamma$-phase, is mostly an inactive form, it is considered that it does not affect efficacy or toxicity [9]. Giacchetti, et al. [17] reported that the toxicity and efficacy of oxaliplatin were not related to the AUC of free platinum, but that hematological toxicity was related to free platinum $\mathrm{C}_{\max }$. Even in our patient, although AUC was slightly high despite a comparable $C_{\text {max }}$ versus normal renal function, no obvious increase in adverse events was seen compared with previous studies $[8,18]$. Given the increase in AUC caused by the second peak, some authors have suggested that administration interval should be extended in consideration of toxicity $[12,13,19]$. On the contrary, we do not consider that extension is necessary because the second and third peaks of free platinum largely consist of inactive forms. Indeed, our study dose was administered safely, albeit that we did not confirm the relationship between free platinum $\mathrm{C}_{\max }$ and toxicity. The possibility that oxaliplatin doses should be further increased until the maximum tolerable $C_{\max }$ is reached may be worth considering.

The timing of hemodialysis requires an interval to allow sufficient distribution to tissue after oxaliplatin infusion in order for the drug to elicit its effect. If hemodialysis is performed immediately after the end of oxaliplatin infusion, the increase in $\mathrm{C}_{\text {max }}$ can be suppressed, but oxaliplatin is removed before it is sufficiently distributed. Conversely, when dialysis is started 1.5 hours after the end of infusion, oxaliplatin is thought to be well distributed to the tissues. In our patient, free platinum $\mathrm{C}_{\max }$ with a reduced oxaliplatin dose was equivalent to that in patients with normal renal function when hemodialysis was performed 1.5 hours after the end of oxaliplatin infusion (Table 1).

This study has some limitations. First, the data were derived from a single patient. Additional examination in a large number of patients is required to evaluate the safety and efficacy of this treatment. Second, the optimal dose could not be confirmed. Although our study dose could be given safely, determining the optimal dose requires a dose escalation study, as is commonly performed in early phase trials.

In conclusion, pharmacokinetics data for oxaliplatin in this hemodialysis patient were comparable to those in patients with normal renal function. This modified FOLFOX6 plus bevacizumab regimen every 2 weeks could be safely given in a hemodialysis patient with colon cancer by reducing oxaliplatin dose to $50 \mathrm{mg} / \mathrm{m}^{2}$, provided hemodialysis was performed 1.5 hours after the end of oxaliplatin infusion. Allowing that further studies are necessary to confirm optimal dose and efficacy, we propose that the administration strategy used in our patient is one option that can be safely administered to patients receiving hemodialysis.

\section{Funding}

No funding was received. 


\section{Availability of Data and Materials}

All data generated or analyzed during this study are included in this published article.

\section{Ethics Approval and Consent to Participate}

This prospective study was approved by the Kobe University Hospital Ethics Committee (No.1481) and conducted in accordance with the Declaration of Helsinki. The patient provided written informed consent before study entry.

\section{Patient Consent for Publication}

Not applicable.

\section{Competing Interests}

Naomi Kiyota has received a research grant from Chugai Pharmaceutical Co., Ltd. outside the submitted work. Hironobu Minami has received research grants from Chugai Pharmaceutical Co., Ltd., Yakult Honsha Co., Ltd., and Kyowa Hakko Kirin Co., Ltd. and has received personal fees from Chugai Pharmaceutical Co., Ltd., and Kyowa Hakko Kirin Co., Ltd. outside the submitted work. The other authors declare that they have no conflict of interest for this study.

\section{References}

1. Eggers PW (2011) Has the incidence of end-stage renal disease in the USA and other countries stabilized? Curr Opin Nephrol Hypertens 20: 241-245.

2. Masakane I, Nakai S, Ogata S, Kimata N, Hanafusa N, et al. (2015) An Overview of Regular Dialysis Treatment in Japan (As of 31 December 2013). Ther Apher Dial 19: 540574.

3. Maisonneuve P, Agodoa L, Gellert R, Stewart JH, Buccianti $G$, et al. (1999) Cancer in patients on dialysis for end-stage renal disease: An international collaborative study. Lancet. 354: 93-99.

4. Stewart JH, Vajdic CM, van Leeuwen MT, Amin J, Webster AC, et al. (2009) The pattern of excess cancer in dialysis and transplantation. Nephrol Dial Transplant 24: 32253231.

5. Shebl FM, Warren JL, Eggers PW, Engels EA (2012) Cancer risk among elderly persons with end-stage renal disease: A population-based case-control study. BMC Nephrol 13: 65 .

6. International Agency for Research on Cancer (2012) GLOBOCAN.

7. Schwartzberg LS, Rivera F, Karthaus M, Fasola G, Canon $\mathrm{JL}$, et al. (2014) PEAK: a randomized, multicenter phase II study of panitumumab plus modified fluorouracil, leucovorin, and oxaliplatin (mFOLFOX6) or bevacizumab plus mFOLFOX6 in patients with previously untreated, unresect- able, wild-type KRAS exon 2 metastatic colorectal cancer. $\mathrm{J}$ Clin Oncol 32: 2240-2247.

8. Yamazaki K, Nagase M, Tamagawa H, Ueda S, Tamura T, et al. (2016) Randomized phase III study of bevacizumab plus FOLFIRI and bevacizumab plus mFOLFOX6 as firstline treatment for patients with metastatic colorectal cancer (WJOG4407G). Ann Oncol 27: 1539-1546.

9. Takimoto $\mathrm{CH}$, Graham MA, Lockwood G, Ng CM, Goetz A, et al. (2007) Oxaliplatin pharmacokinetics and pharmacodynamics in adult cancer patients with impaired renal function. Clin Cancer Res 13: 4832-4839.

10. Takimoto $\mathrm{CH}$, Remick SC, Sharma S, Mani S, Ramanathan RK, et al. (2003) Dose-escalating and pharmacological study of oxaliplatin in adult cancer patients with impaired renal function: a National Cancer Institute Organ Dysfunction Working Group Study. J Clin Oncol 21: 2664-2672.

11. Gori S, Lunardi G, Inno A, Magarotto R, Duranti S, et al. (2014) Pharmacokinetics of oxaliplatin in a hemodialyzed patient: chemotherapy dose adjustment and timing of dialysis. Clin Colorectal Cancer 13: 260-263.

12. Horimatsu T, Miyamoto S, Morita S, Mashimo Y, Ezoe Y, et al. (2011) Pharmacokinetics of oxaliplatin in a hemodialytic patient treated with modified FOLFOX- 6 plus bevacizumab therapy. Cancer Chemother Pharmacol 68: 263-266.

13. Katsumata K, Sumi T, Wada T, Mori Y, Hisada M, et al. (2008) Oxaliplatin for metastatic colon cancer in a patient with renal failure. Clin Med Oncol 2: 97-101.

14. Kume M, Yasui $H$, Yoshikawa $Y$, Horinouchi M, Higashiguchi K, et al. (2012) Transient elevation of serum cystatin C concentrations during perioperative cisplatin-based chemotherapy in esophageal cancer patients. Cancer Chemother Pharmacol 69: 1537-1544.

15. VerstraeteS, HeudiO, CailleuxA, Allain P(2001)Comparison of the reactivity of oxaliplatin, pt(diaminocyclohexane) $\mathrm{Cl} 2$ and pt(diaminocyclohexane1)(OH2)2(2+) with guanosine and L-methionine. J Inorg Biochem 84: 129-135.

16. Graham MA, Lockwood GF, Greenslade D, Brienza S, Bayssas M, et al. (2000) Clinical pharmacokinetics of oxaliplatin: a critical review. Clin Cancer Res 6: 1205-1218.

17. Giacchetti S, Bjarnason G, Garufi C, Genet D, lacobelli S, et al. (2006) Phase III trial comparing 4-day chronomodulated therapy versus 2-day conventional delivery of fluorouracil, leucovorin, and oxaliplatin as first-line chemotherapy of metastatic colorectal cancer: the European Organisation for Research and Treatment of Cancer Chronotherapy Group. J Clin Oncol 24: 3562-3569.

18. Hochster HS, Hart LL, Ramanathan RK, Childs BH, Hainsworth JD, et al. (2008) Safety and efficacy of oxaliplatin and fluoropyrimidine regimens with or without bevacizumab as first-line treatment of metastatic colorectal cancer: results of the TREE Study. J Clin Oncol 26: 3523-3529.

19. Watayo Y, Kuramochi H, Hayashi K, Nakajima G, Kamikozuru $\mathrm{H}$, et al. (2010) Drug monitoring during FOLFOX6 therapy in a rectal cancer patient on chronic hemodialysis.Jpn J Clin Oncol 40: 360-364. 